\title{
On Representation of Functions as Convolution Transforms (*).
}

\author{
Z. Ditzian (Alberta, Edmonton, Canada)
}

Summary. - Necessary and sufficient conditions on $f(x)$ to be a convolution-Stieltjes transform of $\alpha(i)$ where $\alpha(t)$ is such that the transform converges absolutely, are given. The above result is given for Convolution transforms with variation diminishing kernels of class $I$.

\section{Introduction.}

For the convolution transform

$$
f(x)=\int_{-\infty}^{\infty} G(x-t) d \alpha(t)
$$

the representation problem is that of establishing necessary and sufficient conditions on $f$, the generating function, so that $\alpha(t)$, the determining function, belongs to a certain class of functions.

In this paper we shall be interested in the convolution transform with variation diminishing kernel $G(t)$. In fact, we shall restrict ourselves to kernels satisfying

$$
\int_{-\infty}^{\infty} G(t) d t=\left[\exp [b s] \prod_{k=1}^{\infty}\left(1-\frac{s}{a_{k}}\right) \exp \left(s / a_{k}\right)\right]^{-1}=\left[E_{G}(s)\right]^{-1}
$$

where $b, a_{k}$ are real and $\sum a_{k}^{-3}<\infty$. (For the most general variation diminishing kernel the right side is multiplied by $\exp \left(c s^{2}\right)$ for some $e \geqslant 0$.)

Representation theorems for the transforms defined by (1.1) and (1.2) were investigated by $I$. I. Hirschuar and $D$. V. WIDDER in several papers which are summarized in [3, Ch. VII]. Additional results in this direction were obtained recently by G. V. BaDaLaJan [1, p. 485] and by D. Leviatan [4]. In these research works the following cases were investigated: (1) $\alpha(t) \in B \cdot V(-\infty, \infty)$ [3, Oh. VII] and [4] (in the later different conditions in case $G(t) \in$ class II are given); $(2) \alpha(t)$ increasing $[3, \mathrm{Ch}, \mathrm{VII}] ;(3) \propto(t)$ increasing and $\alpha(t) \in B \cdot V(-\infty, \infty)[1, \mathrm{p}, 485] ;(4) \alpha(t)=\int^{t} \varphi(u) d u$ where $\varphi(t) \in L_{P}(-\infty, \infty) 1<p \leqslant \infty[3, \mathrm{Ch} . \mathrm{VII}] ;(5) \alpha(0)=\int^{t} \varphi(u) d u$ and $\varphi \in L_{3 z}(-\infty)$ where $L_{M H}(-\infty, \infty)$ is an Orlicz class and $G(x) \in$ class II $[4]$.

(*) Entrata in Redazione il 19 aprile 1971 . 
Except in case (2) no allowance is made for the possibility that $\alpha(t)$ may behave quite badly for large $|t|$ and case (2) is obviously very restricted.

The results of this paper will treat this question for $G(t) \in$ class $I$, that is, when $E_{g}(S)$ has both positive and negative zeros of $\left(E_{G}(S)\right.$ defined by (1.2)). A necessary and sufficient condition for $f(x)$ to be given by an absolutely convergent integral in (1.1), and a result for $\varphi \in L$ - with $G(t)$ as weight will be described in sections 3 and proved later. An interesting result about the dependence of the convergence of (1.1) for a specific $\alpha(t)$ only on the zeros of $E(S)$ mearest to the origin will be obtained in section 2 and used many times though not in its full generality for the representation theorems.

\section{2. - Convergence Theorem.}

For stating the theorems of this section we have to define:

$$
\alpha_{1}(G)=\max _{a_{k}<0}\left[a_{k},-\infty\right] \quad \alpha_{2}(G)=\min _{a_{k}>0}\left[a_{k}, \infty\right]
$$

where $a_{k:}$ and $G$ are related by (1.2). Define $\alpha_{1}(G)+1$ and $\alpha_{2}(G)+1$ as the multiplicity of $\mu_{1}(G)$ and $\mu_{2}(G)$ in (1.2) respectively. Recall that: $G \in$ class I if both $\alpha_{1}(G)$ and $a_{2}(G)$ are finite.

Theorem 2.1. - Assume: (1) $G \in$ class I; (2) $G_{2}$ satisfies (1.2); (3) $\alpha_{1}\left(G_{1}\right) \geqslant \alpha_{1}\left(G_{2}\right)$ and if the equality holds $\mu_{1}\left(G_{1}\right) \geqslant \mu_{1}\left(G_{2}\right) ;(4) \alpha_{2}\left(G_{1}\right) \leqslant \alpha_{2}\left(G_{2}\right)$ and if the equality holds $\mu_{2}\left(G_{1}\right) \geqslant \mu_{2}\left(G_{2}\right)$. Then, for each function $\propto(t)$ which is of bounded variation locally and for which

$$
\int_{-\infty}^{\infty} G_{1}(x-t) d \alpha(t) \text { converges }
$$

also

$$
\int_{-\infty}^{\infty} G_{2}(x-t) d \alpha(t) \text { converges. }
$$

REMaRK 2.1 $a$. - Since $\int_{-\infty}^{\infty} G_{1}(x-t) d \alpha(t)$ converges for all $x$ so will $\int_{-\infty}^{\infty} G_{2}(x, t) d x(t)$ in spite of the fact that $G_{2}$ is not necessarily of class $I$.

REMARK $2.1 b$. - In case $a_{i}\left(G_{1}\right)=\alpha_{i}\left(G_{2}\right)$ and $\mu_{i}\left(G_{1}\right)=\mu_{i}\left(G_{2}\right)$ for $i=1,2$ the convergence of $\int_{-\infty}^{\infty} G_{1}(x-t) d \alpha(t)$ and $\int_{-\infty}^{\infty} G_{2}(x-t) d \alpha(t)$ are equivalent therefore, for $G \in$ class I convergence of $\int_{-\infty}^{\infty} G(x-t) d \alpha(t)$ depends only on the $a_{k}$ 's nearest to the origin from both sides. 
Remark $2.1 c$. - In Theorem 2.1 we deliberately did not make the restriction that $G(t)$ is non-finite.

Proof of Theorem 2.1 - Let us prove our theorem when both the sequence of positive zeros and and that of negative zeros of $E_{\sigma_{1}}(s)$ are different then those of $E_{\sigma_{3}}(s)$. (We assume the subsequences of positive and negative zeros are ordered by magnitude.) Using Lemma $2.1 b$ of $[3$, p. 121] the proof follows if we can show: (1) $\left|G_{2}(x-t)\right| G_{1}(x-t) \mid \leqslant M$. (2) $G_{2}(x-t) / G_{1}(x-t)$ is continuous in $t$. (3) $G_{2}(x-t) \mid$ $\mid G_{1}(x-t)$ has at most finite number of changes of trend in $(-\infty,-A)$ and $(B, \infty)$ for some $A$ and $B$. Condition (2) is obvious and condition (1) follows a well known result $[3$, Th. $2.1, p .108]$. We shall prove (3) for $t \in(-\infty,-A)$. Using the asymptotic formula [3, p. 110] for $G_{1}(t)$ and $G_{2}(t)$ we have

$$
\left.G_{j}^{(n)}(t)=\sum_{i=1}^{m_{i}}\left(\frac{d}{d t}\right)^{n} P_{i, j}(t) \exp \left(A_{i, j} t\right)\right\}+0\left(\exp \left(k_{j} t\right)\right) \quad t \rightarrow \infty, j=1,2
$$

where $A_{i, j}$ is the sequences of negative zeros of $E_{\sigma_{j}}(s) ; P_{i, j}(t)$ a polynomial of degree $n_{i_{j}}$ that equals the multiplicity of $A_{i_{, j}}$ in $E_{\theta_{j}}(s)$ minus one and $A_{m+1,1}<k_{j}<A_{m, j}$; If $\left\{A_{n, j}\right\}$ both are infinite there exists an $m$ for which the first part of the r.h.s. of (2.2) is different $j=1$ and $j=2$, since the sequences $\left\{A_{i_{, k}}\right\}$ and $\left\{\left(A_{i_{2}}\right\}\right.$ are different and we choose $m_{1}=m_{2}$ to be such. If one of the sequences $\left\{A_{m, i}\right\}$ or both are finite we still can express $G(t)$ like $(2.2)$ in which the $m$ may differ but $k_{1}=k_{2}$ and the first part would be different. One now can show that $(d / d t)\left\{G_{2}(t) / G_{1}(t)\right\}$ has no changes of sign in $t>M$ since in $G_{2}^{\prime}(t) G_{1}(t)-G_{1}^{\prime}(t) G_{2}(t)$ the dominant element that $(2.2)$ yield is of a fixed sign for $t>0$. Therefore $G_{2}(-t) / G_{1}(x-t)$ has no change of trend in $(-\infty,-A)$ for some $A$. Similarly $G_{2}(x-t) / G_{1}(x-t)$ has no change of trend in $(B, \infty)$.

To prove our theorem when either the positive or the negative sebsequence of zeros of $E_{\sigma_{3}}(s)$ and $E_{G_{3}}(s)$ are equal we use $G_{3}(t)$ which has different subsequences of zeros but $\alpha_{i}\left(G_{1}\right)=\alpha_{i}\left(G_{1}\right)=\mu_{i}\left(G_{3}\right)$ for $i=1,2$. Using the above $\int_{-\infty}^{\infty} G_{3}(x-t) d \alpha(t)$ converges and again using the above $\int_{-\infty}^{\infty} G_{2}(x-t) d \alpha(t)$ converges.

\section{3. - Representation Theorems for $G(t) \in$ class I.}

Our main result which we shall prove in section 5 is the following:

THEOREM 3.1. - For a given $G(t)$ that belongs to class $\mathrm{I}$, a necessary and sufficient condition for $f(x)$ to be equal to $\int_{-\infty}^{\infty} G(x-t) d \alpha(t)$ where $\alpha(t)$ is locally of bounded variation satisfying $\int_{-\infty}^{\infty} G\left(x_{0}-t\right)|d \alpha(t)| \leqslant M$ is: is $f(x) \in C^{\infty}, f(x)=o\left(\exp \left(\alpha_{1} x\right)\right) x \rightarrow-\infty$,

7 - Annali di Matematica 
$f(x)=o\left(\exp \left(\alpha_{2} x\right)\right) x \rightarrow \infty$ and for $m \geqslant m_{0}$

$$
\int_{-\infty}^{\infty} G\left(x_{0}-t\right)\left|P_{m}(D) f(t)\right| d t \leqslant M+o(1) \quad m \rightarrow \infty
$$

From this theorem one can deduce the following corollaries.

CoRollaky $3.1 a$. - If a point $x_{0}$ for $m \geqslant m_{0}$

$$
\int_{-\infty}^{\infty} G\left(x_{0}-t\right)\left|P_{m}(D) f(t)\right| d t \leqslant K
$$

$f \in C^{\infty} f(x)=o\left(\exp \left(\alpha_{1} x\right)\right) x \rightarrow-\infty$ and $f(x)=o\left(\exp \left(\alpha_{2} x\right)\right) x \rightarrow \infty$ then the exist $\alpha(t)$ locally of bounded variation such that $f(x)$ is equal to $\int_{-\infty}^{\infty} G(x-t) d \alpha(t)$ which converges absolutely for all $x$.

CoRollarT 3.1 b. - In Corollary $3.1 a$ condition (3.2) can be replaced by for $m \geqslant m_{0}$

$$
\left\{\begin{array}{l}
\int_{0}^{\infty} t^{\mu_{2}} \exp \left(-\alpha_{2} t\right)\left|P_{m}(D) f(t)\right| d t \leqslant L \\
\int_{-\infty}^{0}(-t)^{\mu_{1}} \exp \left(\alpha_{1} t\right)\left|P_{m}(D) f(t)\right| d t \leqslant L
\end{array}\right.
$$

where $\mu_{i}=\mu_{i}(G)$ and $\alpha_{i}=\alpha_{i}(G), i=1,2$.

The following representation theorem concerning determining functions in $L_{P}$ with weight $G\left(x_{0}-t\right)$ would be proved in section 6 .

THEOREM 3.2. - For a given $G(t), G(t) \in$ class $I$, a necessary and sufficient condition for $f(x)$ to be euqal to $\int_{-\infty}^{\infty} G(x-t) \varphi(t)$ where $\int_{-\infty}^{\infty} G\left(x_{0}-t\right)|\varphi(t)|^{p} d t \leqslant M, 1<p<\infty$ is that $f(x) \in C^{\infty} f(x)=o\left(\exp \left(\alpha_{1} x\right)\right) x \rightarrow-\infty f(x)=o\left(\exp \left(\alpha_{2} x\right)\right) x \rightarrow \infty$ and for $m \geqslant m$

$$
\int_{-\infty}^{\infty} G\left(x_{0}-t\right)\left|P_{m}(D) f(t)\right|^{p} d t \leqslant M+o(1) \quad m \rightarrow \infty .
$$

REMARK 3.2 a. - Obviously the analogue Theorem 3.2 for $p=\infty$ is not valid since if $\sup _{t} G\left(x_{0}-t\right)|\varphi(t)| \leqslant M \int_{-\infty}^{\infty} G\left(x_{0}-t\right) \varphi(t) d t$ does not necessarily converge.

The classes of determining functions in the new representation theorems are, as is easily seen, wider then the classes represented before. For instance for every 
$G(t)$ for which $E_{a}(s)=\prod_{k=1}^{\infty}\left(1-s^{2} / a_{n}^{2}\right)$ every polynomial $p(x)$ can be represented $p(x)=\int_{-\infty}^{\infty} G(x-t) d \alpha(t)$ by the theorems above, while $x^{2}$ already is not representable by any of the representation theorems known before.

\section{4. - Some preliminary results.}

We recall, for $f(x)$ defined by (1.1), that

$$
P_{m}(D) f(x)=\int_{-\infty}^{\infty} G_{m}(x-t) d \alpha(t)
$$

where $G_{m}(t)$ satisfies

$$
\int_{-\infty}^{\infty} \exp (-s t) G_{m}(t) d t=\left[E_{m}(s)\right]^{-1} \equiv\left[\exp \left(b_{m} s\right) \prod_{k=m+1}^{\infty}\left(1-\frac{s}{a_{k}}\right) \exp \left(s / a_{k}\right)\right]^{-1}
$$

and where $b_{m}=o(1) m \rightarrow \infty$.

We can obtain now the following result:

LEMma 4.1. - Let $G(t) \in$ class I and $\int_{-\infty}^{\infty} G(x-t) d \alpha(t)$ converges absolutely then

$$
\int_{-\infty}^{\infty} G(x-t) \int_{-\infty}^{\infty} G_{m}(t-v) d \alpha(v)
$$

converges absolutely for $m \geqslant m_{\mathbf{0}}$.

Proof. - We choose $m_{0}$ so that for $k>m_{0}, a_{k} \neq \alpha_{1}(G)$ and $a_{k} \neq \alpha_{2}(G)$. The integral

$$
\int_{-\infty}^{\infty}\left\{\int_{-\infty}^{\infty} G(x-t) G_{m}(t-v) d t\right\}|d \alpha(v)| \equiv \int_{-\infty}^{\infty} \mathfrak{G}(x-v)|d \alpha(v)|
$$

converges using Theorem 2.1, whenever $\int_{-\infty}^{\infty} G(x-t)|d \alpha(v)|$ converges. Therefore, using FUBINI's Theorem (4.3) converges absolutely.

Lemma 4.2. - Suppose: (1) $\psi(t) \in C^{\infty}$. (2) $G(t) \in$ class II. (3) $\mathscr{\Theta}(t)$ is finite or infinite satisfying $\alpha_{i}(\mathcal{G})=\alpha_{i}(G) \equiv \alpha_{i}$ and $\mu_{i}(\mathcal{S})=\mu_{i}(G)$ for $i=1,2$. (4) $f(x)=\int_{-\infty}^{\infty} \mathcal{G}(x-t) \psi(t) d t$. 
Then

$$
\left|\int_{-\infty}^{\infty} G\left(x_{0}-t\right) \psi^{(k)}(t) d t\right| \leqslant M(k)
$$

implies

$$
f^{(k)}(x)=o\left(\exp \left(\alpha_{2} x\right)\right) \quad x \rightarrow \infty \quad f^{(k)}(x)=o\left(\exp \left(\alpha_{1} x\right) \quad x \rightarrow-\infty\right.
$$

Remarque 4.2. - We shall need the above Lemma only for the case $\mathbb{G}$ is finite.

Proof. - Using Lemma 2.1, $I(k, x) \equiv \int_{-\infty}^{\infty} \mathfrak{G}(x-t) \varphi^{(k)} d t$ converges. Integrating by parts and using $(4.5) I(k, x)=f^{(k)}(x)$. We complete our proof using a theorem by $I$. 1. Hirschman and D. V. Wrdder [3, Th. $2.1 A$ p. 147].

Lemnra 4.3. - If $G(t) \in$ class I (finite or infinite) and

$$
\int_{-\infty}^{\infty} G\left(\infty_{0}-t\right)|d \alpha(t)| \leqslant M
$$

then

$$
|f(x)| \leqslant M \operatorname{Max}\left(\exp \left(\alpha_{2}\left(x-x_{0}\right)\right), \exp \left(\alpha_{1}\left(x-x_{0}\right)\right)\right)
$$

where $f(x)=\int_{-\infty}^{\infty} G(x-t) d \alpha(t)$

REMark 4.3 a. - Though this lemma seems weaker then Th. 2.1 of [3, p. 147], replacing $0\left(\exp \left(\alpha_{2} x\right)\right)$ by $M \exp \left(-\alpha_{2} x\right) \exp \left(\alpha_{2} x\right)$ as $x \rightarrow \infty$ for instant, it has the advantage that the estimate (4.8) is given for all $x$ and that it depends only on the constant $M$ in $(4.7)$ and not on the particular $\alpha(t)$.

Proof. - We write

$$
\begin{aligned}
|f(x)| \leqslant \int_{-\infty}^{\infty} G(x-t)|d \alpha(t)|=\int_{-\infty}^{\infty}\left\{\frac{G(x-t)}{G\left(x_{0}-t\right)}\right\} G\left(x_{0}-t\right)|d \alpha(t)| \leqslant \\
\quad \leqslant \max _{i}\left(\frac{G(x-t)}{G\left(x_{0}-t\right)}\right) \int_{-\infty}^{\infty} G\left(x_{0}-t\right)|d \alpha(t)| \leqslant M \cdot \sup _{t}\left(\frac{G(x-t)}{G\left(x_{0}-t\right)}\right) .
\end{aligned}
$$

Recalling

$$
\sup _{t} \frac{G(x-t)}{G\left(x_{0}-t\right)}= \begin{cases}\exp \left(\alpha_{2}\left(x-x_{0}\right)\right) & x \geqslant x_{0} \\ \exp \left(\alpha_{1}\left(x-x_{0}\right)\right) & x<x_{0}\end{cases}
$$

we complete the proof.

We shall also need the following theorem proved in [4]. 
Theorem 4.4. - Let $b_{m}=0$ then

$$
G_{m}(t) \leqslant A S_{m}^{-\frac{1}{2}} \exp \left(-\frac{1}{4} S_{m}^{-1} /|t|\right)
$$

where $\mathbb{S}_{m}=\sum_{k=m+1}^{\infty} a_{k}^{-2}$ and $G_{m}(t)$ is defined by $(4.2)$.

\section{5. - Proof of the main representation Theorem.}

Proof of Theorem 3.1. - First we shall prove (3.1) is necessary. We write using Lemma 4.1 (twice)

$$
\begin{aligned}
& \int_{-\infty}^{\infty} G\left(x_{0}-t\right) d t \int_{-\infty}^{\infty} G(t-v)|d \alpha(t)|=\int_{-\infty}^{\infty} \int_{-\infty}^{\infty} G\left(x_{0}-t\right) G_{m}(t-v) d t|d \alpha(v)|= \\
& =\int_{-\infty}^{\infty} \int_{-\infty}^{\infty} G_{m}\left(x_{0}-t\right) G(t-v) d t|d \alpha(v)|=\int_{-\infty}^{\infty} G_{m}\left(x_{0}-t\right) d t \int_{-\infty}^{\infty} G(t-v)|d \alpha(v)| .
\end{aligned}
$$

Since $\int_{-\infty}^{\infty} G(t-v)|d \alpha(v)|$ is continuous and $\int_{-\infty}^{\infty} G\left(x_{6}-v\right)|d \alpha(v)| \leqslant M$ there exist $\delta_{n}>0$ such that for $\left|x_{\mathrm{c}}-t\right|<\delta_{n} \int_{-\infty}^{\infty} G(t-v) \mid d \alpha(v) \leqslant M^{-\infty}+1 / n$. For those $\delta_{n}$ and any $m$ we have
for all $m$

$$
\int_{x_{0}-\delta_{n}}^{x_{0}+\delta_{n}} G_{m}\left(x_{0}-t\right) \int_{-\infty}^{\infty} G(t-v)|d \alpha(=)| \leqslant M+\frac{1}{n}
$$

For all $t$ the estimate

$$
\int_{-\infty}^{\infty} G(t-v)|d \alpha(v)| \leqslant K \max \left(\exp \left(\alpha_{2} t\right), \exp \left(\alpha_{1} t\right)\right)
$$

follows [3, p. 147]. To avoid awkwardness of expressions we take $b_{m}=0$ the general case is essentially not different. We estimate now

$$
\left\{\int_{-\infty}^{x_{0}-\delta_{n}}+\int_{x_{0}+\delta_{n}}^{\infty}\right\} G_{n}\left(x_{0}-t\right) \int_{-\infty}^{\infty} G(t-v)|d x(v)| \leqslant
$$

$\leqslant\left\{\int_{-\infty}^{\delta_{n}}+\int_{\delta_{n}}^{\infty}\right\} A K \cdot S_{m}^{-\frac{1}{2}} \exp \left\{-\frac{1}{4} S_{m}^{-\frac{1}{2}}|u|\right\}\left(\exp \left(\alpha_{1}\left(x_{\mathrm{f}}-u\right)\right)+\exp \left(x_{\theta}-u\right)\right) d u=0(1) \quad m \rightarrow \infty$

for every fixed $n$. For $\varepsilon>0$ we can choose $\delta_{n}$ such that $(1 / n)<\varepsilon / 2$ and choose $m_{0}$ such that for $m \geqslant m_{0}$ and for the $\delta_{n}$ chosen above (5.3) is estimated by $\varepsilon / 2$. This completes the proof of necessity. 
To prove that the condition stated in Theorem 3.1 are sufficient we first show for all $k$

$$
f^{(k)}(x)=o\left(\exp \left(\alpha_{1} x\right)\right) \quad x \rightarrow-\infty \quad f^{(k)}(x)=o\left(\exp \left(\alpha_{2} x\right)\right) \quad x \rightarrow \infty
$$

We set in Lemma 4.1 $\psi(t)=P_{m_{\mathrm{o}}}(D) f(t)$, obviously $\psi(t) \in C^{\infty}$. The absolute convergence of $\int_{-\infty}^{\infty} G(x-t)\left\{\boldsymbol{P}_{m}(D) f(t)\right\} d t$ for $m \geqslant m_{0}$ at $x=x_{0}$ implies it absolute convergence for all $x^{-\infty}$ and $m \geqslant m_{0}$. Since $P_{m}(D) f(t)=\prod_{k=m_{a}+1}^{m}\left(1-a_{k}^{-1} D \exp \left(a_{k}^{-1} D\right) \psi(t)\right.$ we have

$$
\int_{-\infty}^{\infty} G(x-t) P_{m}(D) f(t) d t=\int_{-\infty}^{\infty} G\left(x-\sum_{k=m_{0}+1}^{m} a_{k}^{-1}-t\right) \prod_{k=m_{0}+1}^{m}\left(1-a_{k}^{-1} D\right) \psi(t) d t
$$

and therefore $\int_{-\infty}^{\infty} G\left(x_{0}-t\right) \psi_{\infty}^{(k)}(t) d t$ converges absolutely. Define $f_{*}(x)=\int_{-\infty}^{\infty} \mathfrak{G}(x-t) \psi(t) d t$ where $\mathfrak{S}(t)$ is defined by $\int_{-\infty}^{\infty} \mathfrak{S}(t) \exp (-s t) d t=\left[P_{m_{\mathrm{o}}}(s)\right]^{-1}$. We shall complete the proof of (5.4) if we show that $f_{*}(x)=f(x)$ since $f_{*}$ satisfies (5.4) by Lemma 4.2. We know that $P_{m_{\theta}}(D)\left[f_{*}(x)-f(x)\right]=0$ but

$$
f_{*}(x)-f(x)=o\left(\exp \left(\alpha_{1} x\right)\right) \quad x \rightarrow-\infty \quad f_{*}(x)-f(x)=o\left(\exp \left(\alpha_{2} x\right)\right) \quad x \rightarrow \infty
$$

and therefore $f_{*}(x)-f(x)=0$.

Define

$$
\alpha_{n}(t)=\int_{0}^{t} P_{n}(D) f(u) f(u) d u
$$

Using the above and Theorem 3.1 of $[3, p .150]$ that

$$
f(x)=\int_{-\infty}^{\infty} H_{n}(x-u) d \alpha_{n}(u)
$$

where $\int_{-\infty}^{\infty} H_{n}(x) \exp (-s x) d x=\left[P_{m}(s)\right]^{-1}$ and $\alpha_{n}(u)=\int_{0}^{u} P_{m}(D) f(t) d t$. We write now

$$
\begin{aligned}
I_{n}(x)-f(x) \equiv \int_{-\infty}^{\infty} G(x-u) d \alpha_{n}(u)-f(x) & =\int_{-\infty}^{\infty}\left[G(x-u)-H_{n}(x-u)\right] d \alpha_{n}(u)= \\
& =\int_{-\infty}^{\infty}\left\{\int_{-\infty}^{\infty}\left[G^{*}(x-v)-H_{n}^{*}(x-v)\right] \Theta(v-u) d v\right\} d \alpha_{n}(u)
\end{aligned}
$$


where $G^{*}(t), G_{n}^{*}(t)$ and $G(t)$ are given by

$$
\begin{aligned}
& \int_{-\infty}^{\infty} G(t) \exp (-s t) d t=\left[\left(1-\alpha_{1}^{-1} s\right){ }^{1+1}\left(1-\alpha_{2}^{-1} s\right)^{\mu_{2}+1}\right]^{-1} \equiv P(s)^{-1} \\
& \int_{-\infty}^{\infty} G^{*}(t) \exp (-s t) d t=E_{G}(s)^{-1} P(s) \equiv E^{*}(s)^{-1}
\end{aligned}
$$

and

$$
\int_{-\infty}^{\infty} H_{n}^{*}(t) \exp (-s t) d t=P_{n}(s)^{-1} P(s) \equiv P_{n}^{*}(s)^{-1}
$$

Using Lemma 4.3 have

$$
\left|\int_{-\infty}^{\infty} \mathcal{G}(v-u) d x_{n}(u)\right| \leqslant M_{1} \max \left(\exp \left(\alpha_{2}\left(v-x_{0}\right)\right), \exp \left(\alpha_{1}\left(v-x_{0}\right)\right)\right) .
$$

Now, we shall prove

$$
\left|I_{n}(x)-f(x)\right| \leqslant o(1)\left(\exp \left(\alpha_{1} x\right)+\exp \left(\alpha_{2} x\right)\right) \quad n \rightarrow \infty
$$

or $\left|I_{n}(x)-f(x)\right|=o(1) \quad n \rightarrow \infty$ uniformly on every compact, and differ by $o(1)\left(\exp \left(\alpha_{2} x\right)+\left(\exp \left(\alpha_{1} x\right)\right) n \rightarrow \infty\right.$ from 0 as $|x| \rightarrow \infty$.

To prove (5.8) we write

$$
\begin{aligned}
\left|I_{n}(x)-f(x)\right| \leqslant M_{2} \int_{-\infty}^{\infty}\left|G^{*}(x-v)-H_{n}^{*}(x-v)\right| & {\left[\exp \left(\alpha_{2} v\right)+\exp \left(\alpha_{1} v\right)\right] d r=} \\
= & \sum M_{2} \exp \left(\alpha_{i} x\right) \int_{-\infty}^{\infty}\left|G^{*}(u)-H_{n}^{*}(u)\right| \exp \left(-\alpha_{i} u\right) d u
\end{aligned}
$$

If we can show now that

a)

$$
\int_{-\infty}^{\infty}\left|G^{*}(u)-H_{n}^{*}(u)\right| \exp \left(-\alpha_{i} u\right) d u=o(1) \quad n \rightarrow \infty i=1,2 ;
$$

we obtain $\left|I_{n}(x)-f(x)\right|=o(1)\left(\exp \left(\alpha_{1} x\right)+\exp \left(\alpha_{2} x\right)\right) n \rightarrow \infty$ It is enough to prove $(a)$ for $i=1$ since the proof for $i=2$ is similar. We choose $R$ independent of $n$ for $n \geqslant n_{0}$ such that

b)

$$
\left\{\int_{-\infty}^{-n}+\int_{n}^{\infty}\right\}\left|G^{*}(u)-H_{n}^{*}(u)\right| \exp \left(-\alpha_{1} u\right) d u<\frac{\varepsilon}{2}
$$


(That this choice is possible we shall prove later). Recalling now that in any finite interval and in particular in $[-R, R]$ and for any $\varepsilon_{1}, \varepsilon_{1}>0$ there exists $n_{1}=n_{1}$. $\cdot\left(\varepsilon_{1}, R\right)$ such that for $n \geqslant n_{1} \geqslant n_{0}\left|G^{*}(u)-H_{n}^{*}(u)\right|<\varepsilon_{1}$. Choosing $\varepsilon / 2=2 R \varepsilon_{1} \exp \left(-\alpha_{1} R\right)$ we observe that $(b)$ would imply $(a)$. To prove $(b)$ we recall that the zeros of $E^{*}(s)$ (and $P_{n}^{*}(s)$ ) are not in $\left[\alpha_{1}, \alpha_{2}\right]$ and therefore by the well known Theorem 2.1 of $\left[3\right.$, p. 108] on asymptotic behaviour of $G(t)$ there exists a constant $R_{0}>0$ such that for $R>R_{0}$

c)

$$
\left\{\int_{-\infty}^{-R}+\int_{\pi}^{\infty}\right\} G^{*}(u) \exp \left(-\alpha_{1} u\right) d u<\frac{\varepsilon}{4}
$$

Following [3, p. 108] and choosing $\beta_{1}<\alpha_{1}<0$ such that $\beta_{1}>a_{t}^{*}$ for all negative $a_{k}^{*}$ for which $E^{*}\left(a_{k}^{*}\right)=0$ we have for $n \geqslant 2$

d)

$$
H_{n}^{*}(u)=\frac{1}{2 \pi i} \int_{-i^{\infty}}^{i^{\infty}} \frac{\exp (s u)}{P_{n}^{*}(s)} d s=\frac{1}{2 \pi i} \int_{\beta_{1}-i^{\infty}}^{\beta_{1}+i^{\infty}} \frac{\exp (s u)}{P_{n}^{*}(s)} d s .
$$

Therefore

$$
H_{n}^{*}(u) \leqslant \frac{1}{2 \pi} \exp \left(\beta_{1} u\right) \int_{-\infty}^{\infty}\left|P_{n}\left(\beta_{1}+i y\right)\right|^{-1} d y \quad u>0 .
$$

but

$$
\left|P_{n}\left(\beta_{1}+i y\right)\right| \geqslant\left|\prod_{i=1}^{2}\left(1-\frac{\beta_{1}+i y}{a_{i}}\right) \exp \left(\beta_{1} / a_{i}^{*}\right)\right| \prod_{i=3}^{n}\left(1-\frac{\beta_{1}}{a_{1}^{*}}\right) \exp \left(\beta_{1} / a_{i}^{*}\right)
$$

and since $\prod_{i=3}^{\infty}\left(1-\beta_{1} / a_{k}^{*}\right) \exp \left(\beta_{i} / a_{k}^{*}\right)$ converges, $\left|\prod_{i=3}^{n}\left(1-\beta_{1} / a_{i}^{*}\right) \exp \left(\beta_{i} \mid a_{k}^{*}\right)\right|>K$ for all $n$ which implies $H_{n}^{*}(u) \leqslant K_{1} \exp \left(\beta_{1} u\right)$ for all $n \geqslant 2$. Therefore, there exists $R$ such that for

$$
n \geqslant 2 \int_{n}^{\infty} H_{n}^{*}(u) \exp \left(-\alpha_{1} u\right) d u<\frac{\varepsilon}{8}
$$

Similarly (even simpler)

$$
\int_{-\infty}^{-R} H_{n}^{*}(u) \exp \left(-\alpha_{1} u\right) d u<\frac{\varepsilon}{8}
$$

Combining the above $\left|I_{n}(x)-f(x)\right|=o(1)\left(\exp \left(\alpha_{1} x\right)+\exp \left(\alpha_{2} x\right)\right) n \rightarrow \infty$. fact

Define now $\beta_{n}(t)=\int_{0}^{t} G\left(x_{0}-u\right) d \alpha_{n}(u)$ it is obvious that $\beta_{n}(t) \in B \cdot V(-\infty, \infty)$ in

$$
\int_{-\infty}^{\infty}\left|d \beta_{n}(t)\right|=\int_{-\infty}^{\infty}\left|d\left\{\int_{0}^{t} G\left(x_{0}-u\right) d \alpha_{n}(u)\right\}\right|=\int_{-\infty}^{\infty} G(x-t)\left|d a_{n}(t)\right| \leqslant M+\varepsilon .
$$


Therefore for all $\psi \in C(\infty, \infty)$ such that both $\lim _{t \rightarrow-\infty} \psi(t)$ and $\lim _{t \rightarrow+\infty} \psi(t)$ exist, we have using Theorem 5.1 of $[3$, p. 156]

$$
\lim _{i \rightarrow \infty} \int_{-\infty}^{\infty} \psi(t) d \beta_{n_{i}}(t)=B_{1} \psi(-\infty)+B_{2} \psi(+\infty)+\int_{-\infty}^{\infty} \psi(t) d \beta(t)
$$

where $\int_{-\infty}^{\infty} \mid d \beta(t) \leqslant M+\varepsilon$ (for all $\varepsilon$ ). Write for $\psi(t)=G(x-t) / G\left(x_{0}-t\right)$ for which $\psi(-\infty)=\exp \left(\alpha_{1}\left(x-x_{0}\right)\right.$ and $\psi(\infty)=\exp \left(\alpha_{2}\left(x-x_{0}\right)\right.$. For that $\psi(t)$ the left side of (5.9) is $\lim I_{n_{i}}(x)$ where $I_{n_{1}}(x)$ is defined in (5.8) which is $o\left(\exp \left(\alpha_{1} x\right)+\exp \left(\alpha_{2} x\right)\right)$ as $|x| \rightarrow \infty, n_{i} \rightarrow \infty$.

Defining $\alpha(t)=\int_{0}^{t} d \beta(t) / G(x-t)$ we have

$$
\int_{-\infty}^{\infty} G\left(x_{0}-t\right)|d \alpha(t)| \leqslant M \quad \text { and } \quad f_{*}(x)=\int_{-\infty}^{\infty} \frac{G(x-t)}{G_{0}(x-t)} d \beta(t)=\int_{-\infty}^{\infty} G(x-t) d \alpha(t) .
$$

Therefore $f_{*}(x)=o\left(\exp \left(\alpha_{1} x\right)\right) x \rightarrow \infty, f^{*}(x)=o\left(\exp \left(\alpha_{2} x\right)\right) x \rightarrow \infty$ and $B_{1}-B_{2}=0$. This implies $f_{*}(x)=f(x)$.

Q.E.D.

\section{6. - Proof of the $L_{v}(G)$ representation Theorem.}

Proof of Theorem 3.2. - First we shall prove that the conditions mentioned are necessary. We write

$$
\begin{aligned}
I(m)=\int_{-\infty}^{\infty} G\left(x_{0}-t\right)\left|P_{m}(D) f(t)\right|^{p} d t=\int_{-\infty}^{\infty} G\left(x_{0}-t\right) d t \mid & \left.\int_{-\infty}^{\infty} G_{m}(t-u) \varphi(u) d u\right|^{p} \leqslant \\
& \leqslant \int_{-\infty}^{\infty} G\left(x_{0}-t\right) d t \int_{-\infty}^{\infty} G_{m}(t-u)|\varphi(u)|^{p} d u .
\end{aligned}
$$

Using Lemma 4.1 and the method of proof of Th. 3.1, we have

$$
I(m) \leqslant \int_{-\infty}^{\infty} G_{m}\left(x_{0}-t\right) d t \int_{-\infty}^{\infty} G(t-u)|\varphi(u)|^{p} d u \leqslant M+\varepsilon .
$$

We shall prove now sufficiency of the conditions in our theorem. The condition

$$
\int_{-\infty}^{\infty} G\left(x_{0}-t\right)\left|P_{m}(D) f(t)\right|^{p} d t \leqslant M+\varepsilon \quad \text { implies } \int_{-\infty}^{\infty} G\left(x_{0}-t\right)\left|P_{m}(D) f(t)\right| d t \leqslant M+1+\varepsilon
$$


since $\left|P_{m}(D) f(t)\right| \leqslant\left|P_{m}(D) f(t)\right|^{p}+1$. This enables us to use the same technique we used in proving Theorem 3.1 to show that

$$
f(x)=\int_{-\infty}^{\infty} H_{m}(x-t) P_{m}(D) f(t) d t
$$

and

$$
\left|f(x)-\int G(x-t) P_{m}(D) f(t) d t\right|=o(1)\left(\exp \left(\alpha_{1} x\right)+\exp \left(\alpha_{2} x\right)\right) \quad m \rightarrow \infty .
$$

Condition (3.4) of our Theorem implies that $P_{m}(D) f(t)$ are in a ball of radius $M-\vdash \varepsilon$ in $L_{p}$ with the weight function $G\left(x_{0}-t\right)$ provided $m \geqslant m_{0}$. This implies there exist $\varphi(t) \in L_{p}\left(G\left(x_{0}-t\right)\right)$ and a subsequence $P_{m_{1}}(D) f(t)$ of $P_{m}(D) f(t)$ such that for all $\psi(t) \in L_{a}\left(G\left(x_{0}-t\right)\right), p^{-1}+q^{-1}=1$, we have

$$
\lim _{m_{i} \rightarrow \infty} \int_{-\infty}^{\infty} \psi(t)\left[P_{m_{i}}(D)(t)\right] G\left(x_{0}-t\right) d t=\int_{-\infty}^{\infty} \psi(t) \varphi(t) G\left(x_{0}-t\right) d t
$$

and $\int_{-\infty}^{\infty}|\varphi(t)|^{p} G\left(x_{0}-t\right) d t \leqslant M+\varepsilon$. In fact since $\varphi$ is the weak limit also of subsequences of the above sequence $\varepsilon$ is arbitrary and $\int_{-\infty}^{\infty} \mid \varphi(t)^{p} G\left(x_{0}-t\right) d t \leqslant M$. We sobstitute $\psi(t)=G(x-t) / G\left(x_{0}-t\right)$ this $\psi(t)$ belong to $L_{a}\left(G\left(x_{0}-t\right)\right)$ since it is bounded (for every fixed $x$ ) and we have

$$
f(x)=\lim _{m_{i} \rightarrow \infty} \int_{-\infty}^{\infty} P_{m_{i}}(D)(t) \cdot G(x-t) d t=\int_{-\infty}^{\infty}\left(\frac{G(x-t)}{G\left(x_{0}-t\right)}\right) \varphi(t) G\left(x_{0}-t\right) d t=\int_{-\infty}^{\infty} \varphi(t) G(x-t) d t .
$$

Q.E.D.

\section{REFERENCES}

[1] G. V. BADALAJAN, The application of a convolution transform to the theory of generalized Stieltjes moment problem, Izv. Akad. Nauk SSSR Ser. Mat., Tom. 31 (1967). English translation: Math. USSR-Izvestija, Vol. I (1967), No. 3, pp. 475-514.

[2] Z. Dirzian, Saturation class for inversion of convolution transform. To appear in the Proceeding of the Conference "On construction function theory", held in Budapest, Aug. 5 . Sept. 5.

[3] I. I. HIRschman - D. V. WIDDER, The convolution transform, Princeton University Press, 1955.

[4] D. LEVIATAN, A new approach to representation theory for convolution transforms, to appear in Pacific Journ. of Math. 